\title{
Vida de anaquel de chorizo elaborado con carne de pechugas de pollos suplementados con aceite de orégano
}

\author{
Shelf life of chorizo manufactured with broiler breasts supplemented with oregano oil

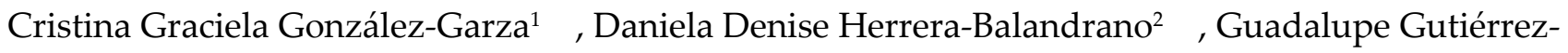 \\ Soto $^{1}{ }^{\circ}$, Carlos Alberto Hernández-Martínez ${ }^{1}$, Emmanuel Flores-Girón ${ }^{3}{ }^{\circledR}$, Armando Quintero- \\ Ramos $^{4}$, Sugey Ramona Sinagawa-García ${ }^{1}$, Gerardo Méndez-Zamora ${ }^{1 *}$
}

${ }^{1}$ Facultad de Agronomía, Universidad Autónoma de Nuevo León, Francisco I. Madero s/n, Ex Hacienda el Canadá, 66050, General Escobedo, Nuevo León, México. ${ }^{2}$ Institute of Agro-Product Processing, Jiangsu Academy of Agricultural Sciences, Nanjing, 210014, China.

${ }^{3}$ Departamento de Ingeniería Agroindustrial, Universidad Autónoma Chapingo, Carretera Federal México-Texcoco, km 38.5, 56230, Texcoco, Estado de México, México.

${ }^{4}$ Facultad de Ciencias Químicas, Universidad Autónoma de Chihuahua, Circuito Universitario s/n, Campus Universitario \#2, 31125, Chihuahua, Chihuahua, México.

*Autor para correspondencia: gerardo.mendezzm@uanl.edu.mx

Fecha de recepción:

5 de febrero de 2020

Fecha de aceptación:

3 de julio de 2020

Disponible en línea:

3 de septiembre de 2020

Este es un artículo en acceso abierto que se distribuye de acuerdo a los términos de la licencia Creative Commons.

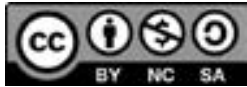

Reconocimiento-

NoComercial-

CompartirIgual 4.0

Internacional

\section{RESUMEN}

Este estudio evaluó chorizos ( $\mathrm{CHO})$ frescos, elaborados con carne de pollos suplementados con aceite de orégano (AO) en su dieta. Se establecieron cinco tratamientos (200, 400, 600, 800 y 1,000 mg kg-1 de AO) y un control. Asimismo, se determinaron el $\mathrm{pH}$, el color, la textura, la capacidad antioxidante, el análisis microbiológico y sensorial. El pH fue mayor $(\mathrm{p}<0.05)$ en el chorizo control, así como cuando contenía $600 \mathrm{mg} \mathrm{kg}^{-1}$ de AO en las evaluaciones de los días 1 y 7. Para el día 7, el color rojo fue significativamente mayor ( $\mathrm{p}>0.05$ ) en los chorizos que contenían 1,000 mg kg-1 de AO, y las bacterias ácido lácticas ( $\mathrm{p}>0.05$ ) mostraron mayor presencia cuando el chorizo contenía $600 \mathrm{mg} \mathrm{kg}^{-1}$ en este mismo periodo. La dureza fue significativamente menor $(p<0.05)$ en el control los días 1 y 7 . Los atributos sensoriales fueron aceptables ( $\mathrm{p}>0.05$ ) en los chorizos con AO. En conclusión, los chorizos de carne de pollo alimentados con $\mathrm{AO}$ mejoraron en $\mathrm{pH}$, color rojo, dureza, bacterias ácido lácticas y aceptabilidad sensorial.

\section{palabras Clave}

Antioxidante, conservación, microbiología, sensorial, textura.

\section{ABSTRACT}

This study evaluated fresh chorizos made of chicken breast meat, with oregano oil (OO) included in the chickens' diet. There were five treatments tested $\left(200,400,600,800\right.$, and $1000 \mathrm{mg} \mathrm{kg}^{-1}$ of OO) and a control, measuring $\mathrm{pH}$, color, texture, antioxidant capacity, microbiologic and sensorial analysis. $\mathrm{pH}$ was higher $(\mathrm{p}<0.05)$ for control chorizo, and when it contained $600 \mathrm{mg} \mathrm{kg}^{-1}$ of $\mathrm{OO}$ at days 1 and 7. 
At day 7, redness was significantly higher $(\mathrm{p}<0.05)$ for chorizos with $1000 \mathrm{mg} \mathrm{kg}^{-1}$ of OO, and lactic acid bacteria ( $\left.\mathrm{p}<0.05\right)$ were mainly present when the chorizo had $600 \mathrm{mg} \mathrm{kg}^{-1}$ at day 7 . Hardness was lower $(\mathrm{p}<0.05)$ for the control group at days 1 and 7. Sensory attributes were fully acceptable $(\mathrm{p}<0.05)$ for chorizos with OO. In conclusion, chorizos made of chicken fed with OO improved their $\mathrm{pH}$, redness, hardness, lactic acid bacteria, and sensory acceptability.

\section{keywords}

antioxidant, conservation, microbiology, sensory, texture.

\section{INTRODUCCIón}

Actualmente, el uso de promotores de crecimiento en la engorda de pollos para la producción de carne destinada al consumo o como materia prima para la elaboración de productos alimenticios ha evidenciado el riesgo hacia la salud de las personas. Debido a esto, la producción y transformación de alimentos busca alternativas viables y óptimas, como la reformulación de los alimentos, la sustitución de ingredientes y, recientemente, el uso de fitobióticos en la engorda de animales, como en los pollos de engorda (Cázares-Gallegos et al. 2019; Hernández-Coronado et al. 2019; Sánchez-Zamora et al. 2019; Silva-Vázquez et al. 2018), lo cual aseguraría la elaboración de productos cárnicos libres de sustancias químicas riesgosas desde el primer eslabón de la cadena agroalimentaria. En este sentido, la industria alimentaria busca contribuir con materia prima producida con aditivos orgánicos y naturales, que no sólo favorezcan el proceso de elaboración de alimentos, sino que también beneficien la salud del consumidor.

Los debates acerca de los efectos en la salud de los productos cárnicos procesados han hecho controversial su producción y consumo (Hung et al. 2016); por ello, investigaciones recientes buscan emplear compuestos naturales en su procesamiento. La adición de antioxidantes naturales en la carne y en productos cárnicos es una de las estrategias en el desarrollo de productos cárnicos saludables y novedosos (Hygreeva et al. 2014; Jiang y Xiong 2016; Charles et al. 2019; Cantú-Valdéz et al. 2020; Herrera-Balandrano et al. 2020). Una opción como condimento son las hojas de orégano y su aceite esencial (AEO), el cual tiene propiedades antioxidantes y antimicrobianas que lo destacan como un conservador natural. Los aceites esenciales son mezclas complejas de líquidos que pueden presentar alta volatilidad, al evaporarse al contacto con el aire, y son obtenidos a partir de diferentes partes de las plantas: flores, semillas, hojas, ramas, corteza, hierbas, madera, frutos y raíces (Burt 2004). Particularmente, el AEO es una sustancia fenólica liposoluble, obtenida por procesos de destilación de las hojas, que contiene carvacrol, timol, $\beta$-mirceno, $\alpha$-terpineno, $\gamma$-terpineno, $p$-cimeno y ceneol (Vazquez y Dunford 2005).

Las especies de orégano más conocidas son Origanum vulgare L. (Lamiaceae), de origen europeo, y Kunth (Verbenaceae), de México (Arcila-Lozano et al. 2004). Actualmente, muchas especias y hierbas, en particular de la familia Lamiaceae, han sido evaluadas como antioxidantes y conservantes en alimentos, lo que incrementa su importancia en la industria alimentaria, por ser una alternativa a los aditivos sintéticos (Dorman et al. 2003). Diversos estudios han sido propuestos sobre la bioactividad del orégano como antioxidante y antimicrobiano (Burt 2004; Fasseas et al. 2007), característica que lo hace viable para usarse en la elaboración de alimentos.

En la actualidad, los nuevos productos cárnicos procesados buscan un posible impacto positivo en la salud, encabezados por la innovación específica y el interés del consumidor, dos características psicológicas personales que forman parte de la intención de compra (Hung et al. 2016). Inclusive, se ha demostrado que el AEO puede sustituir al orégano en polvo (Perales-Jasso et al. 2018) y al vinagre (Charles et al. 2019) en la formulación de chorizo durante 7 y 14 días de almacenamiento, respectivamente.

Por ello, el objetivo del estudio fue investigar el uso de pechuga de pollos suplementados con aceite esencial de orégano en las dietas para elaborar chorizo fresco y evaluar sus propiedades fisicoquímicas, microbiológicas, antioxidantes y sensoriales, así como su textura, en 7 días de conservación. 


\section{Materiales y Métodos}

\section{Diseño experimental}

La engorda de los pollos para obtener la carne (pechuga) fue efectuada en el Campus Marín de la Facultad de Agronomía, Universidad Autónoma de Nuevo León (UANL), en el municipio de Marín, Nuevo León, México. El proceso se llevó a cabo durante 40 días y fue aprobado por el Comité de Cuidado y Bienestar Animal de la UANL.

Un total de 360 pollos Ross308 fueron distribuidos aleatoriamente para seis tratamientos en cinco corrales (12 pollos por corral) cada uno (60 pollos por tratamiento): cinco con aceite esencial de orégano (AEO; Lippia berlandieri Schauer) suministrado (200, 400, 600, 800 y 1,000 mg kg-1 de AEO en las dietas; $60.02 \%$ carvacrol y $23.63 \%$ timol) en las dietas de los pollos y un control (sin AEO) (Cázares-Gallegos et al. 2019). Los pollos fueron alimentados con dieta y agua ad libitum. El AEO se consiguió de la empresa Natural Solutions SMI (Jiménez, Chihuahua, México), y se obtuvo por destilación (arrastre de vapor). El AEO se incorporó mezclándolo con el aceite vegetal de la dieta. Para esta investigación, cinco pollos por corral por tratamiento ( 25 pollos) fueron seleccionados al azar, a una edad de 40 días, para sacrificarlos de acuerdo con la Norma Oficial Mexicana NOM-033-SAG/ZOO-2014 (SAGARPA 2015).

Los códigos establecidos para los chorizos $(\mathrm{CHO})$ elaborados con la carne de pechuga de pollo fueron $\mathrm{CHO0}=$ chorizo con carne control (dieta sin AEO); $\mathrm{CHO} 200$ = chorizo con carne de pollo suplementado con $200 \mathrm{mg} \mathrm{kg}^{-1}$ AEO en dieta; $\mathrm{CHO} 400=$ chorizo con carne de pollo suplementado con $400 \mathrm{mg} \mathrm{kg}^{-1}$ AEO en dieta; $\mathrm{CHO600} \mathrm{=} \mathrm{chorizo} \mathrm{con} \mathrm{carne} \mathrm{de} \mathrm{pollo} \mathrm{su-}$ plementado con $600 \mathrm{mg} \mathrm{kg}^{-1}$ AEO en dieta; $\mathrm{CHO} 800=$ chorizo con carne de pollo suplementado con $800 \mathrm{mg}$ $\mathrm{kg}^{-1}$ AEO en dieta, y CHO1000 = chorizo con carne de pollo suplementado con $1,000 \mathrm{mg} \mathrm{kg}^{-1} \mathrm{AEO}$ en dieta.

\section{Elaboración del chorizo}

Se formularon dos réplicas de $5 \mathrm{~kg}$ de chorizo fresco por tratamiento y consistieron en $75 \%$ carne de pechuga de pollo, $18 \%$ grasa dorsal de cerdo, $2.2 \%$ chile guajillo rojo en polvo, $0.1 \%$ de pimientón en polvo, $0.1 \%$ de orégano en polvo, $0.5 \%$ ajo en polvo, $1.7 \% \mathrm{NaCl}$ y $2.4 \%$ vinagre blanco (Clemente Jacques, Sabormex S.A. de C.V., Ciudad de México, México). La carne y la grasa $\left(4.0^{\circ} \mathrm{C}\right)$ fueron molidas a través de un disco $(9.5 \mathrm{~mm}$; TORREY®, Modelo MV-22R-SS; Grupo Torrey, S.A. de C.V., Nuevo León, México). Después, los ingredientes fueron agregados y mezclados manualmente con la carne y la grasa molidas durante $8 \mathrm{~min}$, adicionando el vinagre lentamente y mezclando durante $5 \mathrm{~min}$, hasta obtener una pasta de chorizo homogénea; la cual fue embutida en una funda artificial de celulosa (3 $\mathrm{cm}$ diámetro), haciendo segmentos de $15 \mathrm{~cm}$ (Charles et al. 2019; González y Díez 2002; Perales-Jasso et al. 2018). El orégano en polvo no fue considerado en la formulación de los tratamientos, para validar el efecto del AEO suplementado en la dieta; así, cualquier efecto en las variables estudiadas sería debido a la carne de pollo, porque las cantidades de los ingredientes fueron exactamente iguales en todos los tratamientos. Los productos elaborados fueron almacenados en refrigeración a $4^{\circ} \mathrm{C}$ durante la experimentación, para medir las variables los días 1 y 7 .

\section{pH y color}

El pHy el color fueron determinados en cinco segmentos de chorizo seleccionados al azar por cada réplica del tratamiento $(n=10)$ en cada día de evaluación. El pH se determinó directamente en el chorizo, utilizando un electrodo de punción (Orion 3 star $^{\mathrm{TM}}$ Thermo Fisher Scientific, Pittsburgh, PA, EUA). Se midió el color con un colorímetro (CR-400 Konica Minolta ${ }^{\mathrm{TM}}$, Tokio, Japón), en la parte interna del chorizo, y éste fue calibrado antes de realizar las pruebas. La luminosidad $\left(\mathrm{L}^{*}\right)$, tendencia al color rojo $\left(\mathrm{a}^{*}\right)$, tendencia al color amarillo $\left(b^{*}\right)$, el ángulo Hue (tonalidad) y Croma (índice de saturación) fueron registrados. Asimismo, se determinaron el cambio de color total $(\triangle \mathrm{CT})$ y el índice de coloración (IC) de acuerdo con Bozkurt y Bayram (2006) y Ledesma et al. (2016).

\section{Análisis de textura}

La fuerza de corte $(\mathrm{FzC})$ y el análisis de perfil de textura (APT) se determinaron con un texturómetro (TA.XT. Plus ${ }^{\circledast}$, Stable Micro Systems Serrey, England). 
La prueba se hizo en diez secciones del chorizo de $30 \mathrm{~mm}$ de diámetro y $45 \mathrm{~mm}$ de largo para $\mathrm{FzC}$, y $30 \mathrm{~mm}$ diámetro y $25 \mathrm{~mm}$ de alto para APT, a una temperatura de $4^{\circ} \mathrm{C}$. La preparación de la muestra, así como la determinación de la $\mathrm{FzC}$ y el APT se realizaron de acuerdo con Perales-Jasso et al. (2018) y Charles et al. (2019). Los parámetros evaluados fueron dureza (Du; g), adhesividad (Ad; $\mathrm{g} \mathrm{s}^{-1}$ ), elasticidad (El; $\mathrm{mm}$ ), cohesividad (Coh; adimensional), gomosidad (Go; g) y masticabilidad (Mas; g mm).

\section{Capacidad antioxidante y análisis microbiológico}

La capacidad antioxidante (CA) en el chorizo de pollo se evaluó mediante la actividad del radical 1, 1-difenil-2-picrilhidrazil (DPPH) de componentes aislados, y se determinó de acuerdo con el método descrito por Mielnik et al. (2003), con las modificaciones establecidas por Perales-Jasso et al. (2018). Los ensayos fueron efectuados en dos réplicas por triplicado $(n=6)$.

El conteo microbiano y la preparación de las muestras se hicieron de acuerdo con la NOM-110SSA1-1994(SS1995), así como con ligeras modificaciones establecidas por Charles et al. (2019) y Perales-Jasso et al. (2018). El conteo de mesófilos y psicrótrofos totales se llevó a cabo en agar cuenta estándar, variando la temperatura de incubación de 37 y $4^{\circ} \mathrm{C}$ por $24 \mathrm{~h}$, respectivamente. El conteo de bacterias ácido lácticas (BAL) fue efectuado en el medio MRS, incubando las placas a $37^{\circ} \mathrm{C}$ por $24 \mathrm{~h}$. El medio violeta rojo y bilis agar (RVBA), utilizado para la determinación de coliformes, se realizó después de $24 \mathrm{~h}$ de incubación de las placas a $37^{\circ} \mathrm{C}$. El medio papa dextrosa agar (PDA) se usó para hongos y levaduras (incubación $37^{\circ} \mathrm{C}$ por $24-48 \mathrm{~h}$ ). El conteo de los microorganismos se llevó a cabo en las dos réplicas por tratamiento por triplicado $(n=6)$. De los Laboratorios CONDA SA (Torrejón de Ardoz, Madrid, España) se obtuvieron los medios de cultivo.

\section{Evaluación sensorial}

A los días 1 y 7, una evaluación sensorial afectiva por atributos se hizo con 30 consumidores semientrenados con pruebas de identificación de olores y sabores. Aproximadamente, $500 \mathrm{~g}$ de chorizo se cocinaron en un sartén a temperatura de $85^{\circ} \mathrm{C}$ durante $10 \mathrm{~min}$., mezclando lento y constante; después, se enfriaron a $28^{\circ} \mathrm{C}$, para su evaluación. Cada consumidor evaluó $10 \mathrm{~g}$ de chorizo cocinado, servidos en vasos de plástico codificados con tres números al azar. Los atributos evaluados fueron color rojo, olor, sabor, blandura y aceptabilidad global. Se utilizó una escala hedónica de siete puntos para evaluar los atributos, donde $7=$ me gusta mucho y 1 = me disgusta mucho (Meilgaard et al. 2006).

\section{Análisis estadístico}

Se analizaron los datos con el procedimiento MIXED de SAS (SAS 2006) y el modelo estadístico: $y_{\mathrm{ij}}=\mu+$ $\tau_{\mathrm{i}}+\delta_{\mathrm{j}}+(T \delta)_{\mathrm{ij}}+\varepsilon_{\mathrm{ij}}$; dónde: $\mathrm{y}_{\mathrm{ijk}}=$ variables evaluadas a través del tiempo; $\mu=$ media general; $T_{i}=$ efecto del i-esimo tratamiento $(\mathrm{CHO} 0-\mathrm{CHO} 1000) ; \delta_{j}=$ efecto del j-esimo día de evaluación $\left(1\right.$ y 7 d); $(T \delta)_{i j}=$ efecto de la interacción entre el i-esimo tratamiento y el j-esimo día; $\varepsilon_{\mathrm{ijk}}=$ error aleatorio distribuido en forma normal con media y varianza $\left[\varepsilon_{i j} \sim N\left(\mu, \sigma^{2}\right)\right]$. Cuando existió efecto estadístico $(\mathrm{p}<0.05)$ en las variables, la prueba estadística Tukey fue usada para la comparación de medias.

\section{Resultados Y Discusión}

\section{pH y color}

El pH y el color son dos parámetros determinantes en la evaluación de la calidad fisicoquímica de los alimentos durante su almacenamiento. Hasta el momento no existen antecedentes de investigaciones que involucren la elaboración de chorizo con carne de pollos alimentados con dietas suplementadas con AEO. Los chorizos elaborados con la pechuga de pollo presentaron diferencias significativas $(\mathrm{p}<0.05)$ en $\mathrm{pH}$ y color durante el almacenamiento (Cuadro 1). Del día 1 al 7 el pH aumentó en el chorizo control (CHO0), pero decreció en el chorizo $\mathrm{CHO} 400$. Los valores de $\mathrm{pH}$ al día 1 fueron similares a los de la salchicha fermentada con bacterias probióticas -estudio efectuado por Radulović et al. (2011) - , aunque al día 7 los chorizos elaborados con carne de pollo presentaron $\mathrm{pH}$ más alto. Estos resultados indican que la maduración de los chorizos elaborados se ve favorecida por el uso de carne de pollos suplementados con AEO, el cual provoca 
un descenso del pH. Corral et al. (2013) mencionaron que bajos niveles de $\mathrm{a}_{\mathrm{w}} \mathrm{y}$ pH garantizan la estabilidad y calidad de los embutidos, y que la caída del $\mathrm{pH}$ hasta niveles cercanos a 4.5 en salchichas de fermentación lenta se debe al crecimiento de las bacterias ácido lácticas. Estos autores obtuvieron valores de $\mathrm{pH}$ en salchichas de fermentación lenta a 7 días, similares a los obtenidos en los chorizos de la presente investigación.
Respecto al color, la luminosidad $\left(\mathrm{L}^{*}\right)$ incrementó (p $<0.05$ ) el día 1 para CHO600, CHO800 y CHO1000, y para el día 7, CHO800 fue el más luminoso y CHO200 menos luminoso $(\mathrm{p}<0.05)$. El color rojo $\left(\mathrm{a}^{*}\right)$ no fue diferente $(p>0.05)$ en el día 1 , mientras que para el día $7 a^{*}$ mejoró ( $p$ $<0.05)$ en $\mathrm{CHO} 200, \mathrm{CHO} 400, \mathrm{CHO} 600$ y CHO1000, pero $\mathrm{CHO} 800$ presentó la menor tendencia hacia $\mathrm{a}^{*}$. Respecto al color amarillo $\left(b^{*}\right)$, en el día 1 de evaluación, los chorizos

Cuadro 1. Efecto de la pechuga de pollos alimentados con aceite de orégano sobre el color de los chorizos.

\begin{tabular}{|c|c|c|c|c|c|c|c|c|}
\hline \multirow{2}{*}{$\begin{array}{l}\text { Días/ } \\
\text { Trat }^{1}\end{array}$} & \multicolumn{8}{|c|}{ Variables de color ${ }^{2}$} \\
\hline & $\mathrm{pH}$ & $\mathrm{L}^{*}$ & $a^{*}$ & $b^{*}$ & Hue & Chroma & $\Delta \mathrm{E}$ & IC \\
\hline \multicolumn{9}{|l|}{ Día 1} \\
\hline $\mathrm{CHOO}$ & $5.52 \mathrm{a}$ & $46.14 \mathrm{bc}$ & $31.19 \mathrm{a}$ & $33.33 a$ & $46.78 \mathrm{a}$ & $45.63 a$ & $64.58 \mathrm{a}$ & $161.94 a$ \\
\hline $\mathrm{CHO} 200$ & $5.45 \mathrm{~cd}$ & $45.85 c$ & $31.75 \mathrm{a}$ & $32.03 \mathrm{ab}$ & $45.50 \mathrm{~b}$ & $44.89 \mathrm{a}$ & $64.50 \mathrm{a}$ & $157.23 \mathrm{ab}$ \\
\hline $\mathrm{CHO} 400$ & $5.44 \mathrm{~d}$ & $46.11 b c$ & $31.58 \mathrm{a}$ & $32.34 \mathrm{ab}$ & $45.20 \mathrm{~b}$ & $44.86 a$ & 64.37ab & 157.37ab \\
\hline $\mathrm{CHO} 600$ & $5.48 b$ & $47.46 a$ & $31.75 a$ & $32.39 \mathrm{ab}$ & $45.56 b$ & $45.26 a$ & $63.45 \mathrm{bc}$ & $151.82 b c$ \\
\hline $\mathrm{CHO} 800$ & $5.48 b$ & $47.55 a$ & $31.47 \mathrm{a}$ & $31.74 \mathrm{~b}$ & $45.29 b$ & $44.80 \mathrm{a}$ & $62.96 c$ & $147.94 \mathrm{c}$ \\
\hline CHO1000 & $5.47 \mathrm{bc}$ & $46.99 \mathrm{ab}$ & $31.23 a$ & $31.42 \mathrm{~b}$ & $45.22 b$ & $44.31 \mathrm{a}$ & $63.11 c$ & $148.40 \mathrm{c}$ \\
\hline \multicolumn{9}{|l|}{ Día 7} \\
\hline $\mathrm{CHO} 0$ & $5.47 a$ & $50.24 \mathrm{~b}$ & $29.16 b c$ & $35.21 b c$ & $50.54 a$ & $45.67 \mathrm{~b}$ & $61.52 \mathrm{ab}$ & $150.95 \mathrm{ab}$ \\
\hline $\mathrm{CHO} 200$ & $5.34 \mathrm{c}$ & $48.93 c$ & $30.64 a$ & $33.65 d$ & $47.87 \mathrm{c}$ & $45.52 b$ & $62.41 \mathrm{a}$ & $150.55 \mathrm{ab}$ \\
\hline $\mathrm{CHO} 400$ & $5.32 c$ & $50.29 b$ & $30.18 a b$ & $34.15 \mathrm{~cd}$ & $48.54 c$ & $45.58 b$ & $61.43 a b$ & $146.85 b$ \\
\hline $\mathrm{CHO} 600$ & $5.38 b$ & $51.00 \mathrm{~b}$ & $30.04 a b$ & $35.98 \mathrm{ab}$ & $50.15 \mathrm{ab}$ & $46.87 a$ & $61.78 \mathrm{a}$ & $152.50 \mathrm{a}$ \\
\hline CHO800 & $5.35 \mathrm{bc}$ & $52.20 \mathrm{a}$ & $28.94 \mathrm{c}$ & $36.51 \mathrm{a}$ & $51.49 a$ & $46.65 a$ & $60.69 b$ & 148.86ab \\
\hline CHO1000 & $5.34 \mathrm{c}$ & $50.62 b$ & $30.88 \mathrm{a}$ & $35.31 b$ & $48.82 \mathrm{bc}$ & $46.91 a$ & $62.13 a$ & $151.85 \mathrm{ab}$ \\
\hline EEM & 0.01 & 0.25 & 0.22 & 0.32 & 0.29 & 0.31 & 0.24 & 1.58 \\
\hline \multicolumn{9}{|l|}{ P-value } \\
\hline$\tau_{i}$ & $<0.0001$ & $<0.0001$ & 0.0174 & $<0.0001$ & $<0.0001$ & 0.0623 & 0.0001 & 0.0016 \\
\hline$\delta_{j}$ & $<0.0001$ & $<0.0001$ & $<0.0001$ & $<0.0001$ & $<0.0001$ & $<0.0001$ & $<0.0001$ & 0.0163 \\
\hline$(T \delta)_{\mathrm{ij}}$ & 0.0002 & 0.0345 & 0.0012 & 0.0007 & $<0.0001$ & 0.0009 & 0.0089 & 0.0046 \\
\hline
\end{tabular}

${ }^{1}$ Tratamientos $\mathrm{CHO}$ = chorizo con carne control (dieta sin $\mathrm{AEO}$ ); $\mathrm{CHO} 200$ = chorizo con carne de pollo suplementado con 200 mg kg-1 $\mathrm{AEO}$ en dieta; $\mathrm{CHO} 400$ = chorizo con carne de pollo suplementado con $400 \mathrm{mg} \mathrm{kg}^{-1} \mathrm{AEO}$ en dieta; $\mathrm{CHO} 600$ = chorizo con carne de pollo suplementado con $600 \mathrm{mg} \mathrm{kg}^{-1} \mathrm{AEO}$ en dieta; $\mathrm{CHO} 800$ = chorizo con carne de pollo suplementado con $800 \mathrm{mg} \mathrm{kg}^{-1} \mathrm{AEO}$ en dieta, y CHO1000 = chorizo con carne de pollo suplementado con 1000 mg kg-1 $\mathrm{AEO}$ en dieta; $\mathrm{EEM}=$ error estándar de medias; $\mu=$ media global; $\mathrm{T}_{\mathrm{i}}=$ efecto del i-esimo tratamiento; $\delta_{\mathrm{j}}=$ efecto del j-esimo día de evaluación $(1 \mathrm{y} 7 \mathrm{~d}) ;(\mathrm{T} \delta)_{\mathrm{ij}}=$ efecto de la interacción entre el i-esimo tratamiento y el j-esimo día.

${ }^{2} \mathrm{~L}^{*}=$ luminosidad; $\mathrm{a}^{*}=$ tendencia al color rojo; $\mathrm{b}^{*}=$ tendencia al color amarillo; Hue = ángulo Hue; Chroma = índice de saturación; $\Delta \mathrm{E}=$ cambio de color total; IC = índice de coloración.

a-d Medias $(n=10)$ dentro de la misma columna y para diferentes tratamientos en cada día con diferente superíndice difieren significativamente cuando el p-value de $(T \delta)_{\mathrm{ij}}<0.05$. 
$\mathrm{CHO}$, $\mathrm{CHO} 200, \mathrm{CHO} 400$ y $\mathrm{CHO} 600$ tuvieron la mayor tendencia hacia $\mathrm{b}^{*}$, mientras que $\mathrm{CHO} 800$ y $\mathrm{CHO} 1000$ mostraron la menor $b^{*}$, pero en el día 7, CHO800 mostró la mayor tendencia a $b^{*}$ y CHO200 obtuvo la menor. En el día 1, el chorizo CHO0 obtuvo la mejor tonalidad (Hue) respecto a los otros tratamientos, mientras que para el día 7 , tanto $\mathrm{CHO} 0$ como $\mathrm{CHO} 800$ presentaron una mejora en la tonalidad y CHO200 no mejoró su tono.

Por otra parte, Chroma no fue diferente el día 1 ( $p$ > 0.05), pero para el día 7, CHO600, CHO800 y CHO1000 fueron los de mayor saturación en el color (Chroma). En un estudio de salchichas fermentadas realizado por Menegas et al. (2013), los valores de color fueron menores. Posiblemente estas diferencias se deben a su formulación, ya que estos autores no usaron chile rojo en polvo. Por otra parte, en el día 1 , el cambio de color total $(\Delta \mathrm{E})$ incrementó en $\mathrm{CHO} 0$ y $\mathrm{CHO} 200$, pero decreció en $\mathrm{CHO} 600, \mathrm{CHO} 800$ y CHO1000; mientras que para el día 7, CHO200 presentó más cambio de color y CHO800 menos. En cuanto al índice de coloración (IC), CHO0 aumentó para el día 1, pero disminuyó para el día 7 , mientras que $\mathrm{CHO} 600, \mathrm{CHO} 800$ y CHO1000 fueron los de más IC. Similares resultados de Hue, Chroma, $\Delta E$ e IC fueron obtenidos en salchicha fermentada (Bozkurt y Bayram 2006). Estas diferencias pueden deberse al tipo de carne usada; Bozkurt y Bayram (2006) emplearon carne de cordero, y esta investigación fue con pollo. En otro estudio de chorizo ahumado realizado por Ledesma et al. (2016) durante 11 días se obtuvieron valores similares de color $\left(L^{*}, a^{*}, b^{*}\right.$, Hue, Chroma e IC), cuando evaluaron fundas naturales y sintéticas.

Los valores de $a^{*}$ podrían indicar que la suplementación de AEO en pollos durante su engorda puede beneficiar la conservación del color rojo $\left(a^{*}\right)$ de los chorizos. Lorenzo et al. (2013) obtuvieron resultados similares en $L^{*}, a^{*} y b^{*}$ en chorizo enriquecidos con semilla de uva y extracto de castaña $(1 \mathrm{~g} / \mathrm{kg})$. Estos autores indicaron que, si la formación del color rojo ladrillo se da durante la maduración de los chorizos, la adición de antioxidantes naturales podría proporcionar embutidos más atractivos. En este estudio, el AEO pudo conservar la pigmentación rojiza del chorizo debido al chile rojo en polvo, al interactuar los grupos $\mathrm{OH}$ de los compuestos antioxidantes del AEO (carvacrol y timol). Por otra parte, la adición de cloruro de potasio $(0.64 \%)$ y aceite de girasol (1.5 y
$3 \%$ ) disminuyen el color en salchichas no fermentadas, debido a un alto contenido de grasa (80:20), lo que hace visible una coloración blanca del producto y la pigmentación rojiza de la carne (Mora-Gallego et al. 2016).

\section{Análisis de textura}

El estudio de textura de los alimentos se basa en la fuerza de corte $(\mathrm{FzC})$ y en el análisis del perfil de textura (APT). El Cuadro 2 muestra la FzC y el APT de los chorizos elaborados con carne de pechuga de pollos suplementados con AEO. La FzC, dureza, adhesividad, elasticidad, gomosidad y masticabilidad fueron afectadas por la interacción de tratamientos y días $\left((T \delta)_{i j}<0.05\right)$, mientras que la cohesividad fue afectada por los tratamientos en cada día evaluado $(\mathrm{p}<0.05)$. Para los días 1 y 7, la FzC incrementó en $\mathrm{CHO} 1000$ y decreció para $\mathrm{CHO}$. En cuanto a dureza, los días 1 y 7, CHO800 fue más duro y $\mathrm{CHO} 0$ tuvo menos dureza; asimismo, CHO800 fue más adhesivo los días 1 y 7 , pero CHO600 fue menos adhesivo en el día 1 y $\mathrm{CHO} 0$ en el día 7. Por otro lado, $\mathrm{CHO} 200$ fue más elástico y cohesivo los días 1 y 7 , pero $\mathrm{CHO} 600$ presentó menos elasticidad y cohesividad en el día 1 y $\mathrm{CHO} 0$ en el día 7. Los días 1 y 7, CHO800 mostró más gomosidad y masticabilidad y $\mathrm{CHO} 0$ fue el menos gomoso y masticable.

Escasos estudios han evaluado el comportamiento de la textura en el tiempo de chorizos elaborados con carne de pollo. Los resultados obtenidos en esta investigación contrastan con los obtenidos por Menegas et al. (2013). Estos autores encontraron valores más altos en los parámetros de textura en salchichas de pollo fermentadas en seco. Estas diferencias pudieron deberse a los ingredientes usados en la formulación de los productos, ya que en los chorizos fue usada grasa dorsal de cerdo, la cual aporta más blandura en las pastas cárnicas. Otros autores han obtenido resultados más altos en el análisis de textura (Bozkurt y Bayram 2006; Corral et al. 2013; Ledesma et al. 2016; Lorenzo et al. 2013; Perales-Jasso et al. 2018), porque emplean carne de cerdo, así como por la variación de los ingredientes en su formulación y los periodos evaluados. Este contraste podría deberse a que la carne de cerdo es 
Cuadro 2. Fuerza de corte y análisis del perfil de textura de chorizos elaborados con carne de pollos alimentados con aceite esencial de orégano.

\begin{tabular}{|c|c|c|c|c|c|c|c|}
\hline \multirow{2}{*}{$\begin{array}{l}\text { Días/ } \\
\text { Trat }^{1}\end{array}$} & \multirow[b]{2}{*}{$\mathrm{FzC}\left(\mathrm{g}_{\mathrm{f}}\right)$} & \multicolumn{6}{|c|}{ Análisis de textura $^{2}$} \\
\hline & & $\mathrm{Du}(\mathrm{g})$ & $\begin{array}{l}\text { Ad } \\
\left(\mathrm{g} \mathrm{s}^{-1}\right)\end{array}$ & $\mathrm{El}(\mathrm{mm})$ & Coh & Go (g) & $\begin{array}{l}\text { Mas } \\
(\mathrm{g} \mathrm{mm})\end{array}$ \\
\hline \multicolumn{8}{|l|}{ Día 1} \\
\hline $\mathrm{CHO} 0$ & $131.51 \mathrm{~d}$ & $168.22 \mathrm{c}$ & $-59.81 a b$ & $0.77 \mathrm{ab}$ & $0.55 \mathrm{ab}$ & $92.82 \mathrm{~d}$ & $76.56 b c$ \\
\hline $\mathrm{CHO} 200$ & $208.13 b c$ & $194.99 b c$ & $-78.79 b c$ & $0.79 a$ & $0.58 \mathrm{a}$ & $113.49 \mathrm{bcd}$ & $90.46 \mathrm{ab}$ \\
\hline $\mathrm{CHO} 400$ & $202.49 b c$ & $203.02 b c$ & $-60.33 a b$ & $0.73 a b$ & $0.57 \mathrm{ab}$ & $115.26 \mathrm{bc}$ & $84.49 b c$ \\
\hline $\mathrm{CHO} 600$ & $180.27 \mathrm{c}$ & $175.27 \mathrm{bc}$ & $-49.12 a$ & $0.68 b$ & $0.54 b$ & $94.69 \mathrm{~cd}$ & $64.14 \mathrm{c}$ \\
\hline $\mathrm{CHO} 800$ & $219.97 \mathrm{ab}$ & $262.29 a$ & $-80.09 c$ & $0.74 a b$ & $0.56 \mathrm{ab}$ & $146.53 a$ & $108.99 a$ \\
\hline CHO1000 & $246.47 a$ & $212.97 \mathrm{~b}$ & $-59.44 a b$ & $0.69 \mathrm{ab}$ & $0.55 b$ & $116.85 b$ & $81.02 \mathrm{bc}$ \\
\hline$\mu$ & 198.14 & 202.79 & -64.60 & 0.73 & 0.56 & 113.27 & 84.28 \\
\hline \multicolumn{8}{|l|}{ Día 7} \\
\hline $\mathrm{CHOO}$ & $175.47 \mathrm{c}$ & $552.76 c$ & $-162.61 \mathrm{a}$ & $0.43 c$ & $0.36 b$ & 201.51c & $86.53 c$ \\
\hline CHO2O0 & $265.44 \mathrm{ab}$ & $864.54 \mathrm{ab}$ & $-321.96 b$ & $0.58 \mathrm{a}$ & $0.39 \mathrm{ab}$ & $340.34 \mathrm{ab}$ & $198.01 \mathrm{a}$ \\
\hline $\mathrm{CHO} 400$ & $308.76 \mathrm{ab}$ & $679.70 \mathrm{bc}$ & $-235.64 a$ & $0.56 \mathrm{ab}$ & $0.40 \mathrm{a}$ & $269.24 b c$ & $149.14 \mathrm{ab}$ \\
\hline $\mathrm{CHO} 600$ & $253.72 b$ & $534.04 \mathrm{c}$ & $-191.99 a$ & $0.50 \mathrm{abc}$ & $0.39 \mathrm{ab}$ & $207.64 c$ & $104.89 b c$ \\
\hline CHO800 & $243.71 b$ & 877.90a & $-334.37 b$ & $0.56 a b$ & $0.39 \mathrm{ab}$ & $345.93 a$ & $195.87 a$ \\
\hline CHO1000 & $325.74 a$ & $631.56 \mathrm{c}$ & $-212.52 a$ & $0.48 b c$ & $0.38 \mathrm{ab}$ & $238.25 c$ & $115.08 \mathrm{bc}$ \\
\hline EEM & 12.54 & 33.49 & 15.06 & 0.02 & 0.01 & 12.86 & 9.86 \\
\hline$\mu$ & 262.14 & 690.08 & -243.18 & 0.52 & 0.39 & 267.15 & 141.59 \\
\hline \multicolumn{8}{|l|}{ P-value } \\
\hline$\tau_{i}$ & $<0.0001$ & $<0.0001$ & $<0.0001$ & $<0.0001$ & 0.0011 & $<0.0001$ & $<0.0001$ \\
\hline$\delta_{j}$ & $<0.0001$ & $<0.0001$ & $<0.0001$ & $<0.0001$ & $<0.0001$ & $<0.0001$ & $<0.0001$ \\
\hline$(T \delta)_{i j}$ & 0.0253 & $<0.0001$ & $<0.0001$ & 0.0082 & 0.2467 & $<0.0001$ & $<0.0001$ \\
\hline
\end{tabular}

${ }^{1}$ Tratamientos $\mathrm{CHO}=$ chorizo con carne control (dieta sin $\mathrm{AEO}$ ); $\mathrm{CHO} 200=$ chorizo con carne de pollo suplementado con $200 \mathrm{mg} \mathrm{kg}-1 \mathrm{AEO}$ en dieta; $\mathrm{CHO} 400=$ chorizo con carne de pollo suplementado con $400 \mathrm{mg} \mathrm{kg}-1 \mathrm{AEO}$ en dieta; $\mathrm{CHO} 600=$ chorizo con carne de pollo suplementado con $600 \mathrm{mg}$ kg- $1 \mathrm{AEO}$ en dieta; $\mathrm{CHO} 800=$ chorizo con carne de pollo suplementado con $800 \mathrm{mg}$ kg-1 AEO en dieta; $\mathrm{CHO} 1000=$ chorizo con carne de pollo suplementado con 1000 mg kg- 1 AEO en dieta; $E E M=$ error estándar de medias; $\mu=$ media global; $T$ i= efecto del i-esimo tratamiento; $\delta j=$ efecto del j-esimo día de evaluación $(1$, y 7 d); $(T \delta) i j=$ efecto de la interacción entre el i-esimo tratamiento y el j-esimo día.

${ }^{2} \mathrm{FC}=$ fuerza de corte $(\mathrm{N})$; Dur = dureza $(\mathrm{g})$; Adh = adhesividad; Elast = elasticidad Coh = cohesividad (adimensional); Gom = gomosidad; Mast = masticabilidad. a-d Medias $(n=10)$ dentro de la misma columna y para diferentes tratamientos en cada día con diferente superíndice difieren significativamente cuando el p-value de $(T \delta) i j<0.05$.

más dura que la de pollo, debido a los ácidos grasos presentes en las carnes (Wood et al. 2003), por lo que en un producto elaborado con carne de pollo presentaría una menor dureza. Los incrementos de la fuerza de corte y dureza de los chorizos CHO800 y CHO1000 en los días 1 y 7 pueden ser atribuibles al aceite de orégano, porque éste mejora la conservación por el efecto del carvacrol y timol como antimicrobiano y antioxidante (Perales-Jasso et al. 2018).

\section{Análisis microbiológico y capacidad antioxidante}

El Cuadro 3 presenta los resultados del análisis microbiológico y la capacidad antioxidante de los chorizos elaborados con carne de pollo. Estas variables fueron 


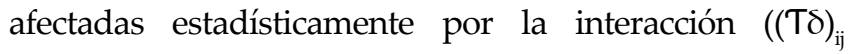
$<0.05$ ) entre los tratamientos ( $\mathrm{Ti}$ ) y los días de evaluación $\left(\delta_{\mathrm{j}}\right)$. Para el día 1, en el chorizo CHO200 incrementó el conteo de mesófilos, en $\mathrm{CHO}$ hubo más psicrótrofos, en CHO800 más coliformes y $\mathrm{CHO} 1000$ resultó tener más BAL; en contraste, los conteos más bajos de estos mi- croorganismos fueron para $\mathrm{CHO} 400, \mathrm{CHO} 600$ y $\mathrm{CHO}$, respectivamente. Para el día 7, CHO0 mostró los conteos más elevados de mesófilos y psicrótrofos, mientras que CHO600 y CHO800 obtuvieron los menores conteos de estas bacterias. Los coliformes no presentaron diferencias significativas $(\mathrm{p}>0.05)$, pero fueron altos

Cuadro 3. Análisis microbiológico y capacidad antioxidante de los chorizos elaborados con carne de pollos suplementados con aceite esencial de orégano.

\begin{tabular}{|c|c|c|c|c|c|}
\hline \multirow{2}{*}{$\begin{array}{l}\text { Días/ } \\
\text { Trat }^{1}\end{array}$} & \multicolumn{4}{|c|}{ Microbiología (log UFC/g)² } & \multirow{2}{*}{$\mathrm{CA}^{3}(\%)$} \\
\hline & Mesófilos & Psicrótrofos & Coliformes & BAL & \\
\hline \multicolumn{6}{|l|}{ Día 1} \\
\hline $\mathrm{CHOO}$ & $7.72 \mathrm{c}$ & $7.87 \mathrm{a}$ & $7.30 \mathrm{c}$ & $8.38 \mathrm{~d}$ & $5.59 \mathrm{a}$ \\
\hline $\mathrm{CHO} 200$ & $7.89 a$ & 7.60ab & $7.36 \mathrm{bc}$ & $8.57 c$ & $4.00 \mathrm{ab}$ \\
\hline $\mathrm{CHO} 400$ & $6.61 \mathrm{e}$ & $7.50 \mathrm{ab}$ & $7.21 \mathrm{~d}$ & $8.63 b$ & $4.19 \mathrm{ab}$ \\
\hline $\mathrm{CHO} 600$ & $6.95 \mathrm{~d}$ & $7.28 b$ & $7.39 \mathrm{ab}$ & $8.67 \mathrm{a}$ & $3.54 \mathrm{~b}$ \\
\hline $\mathrm{CHO} 800$ & $7.74 \mathrm{bc}$ & $7.51 \mathrm{ab}$ & $7.47 \mathrm{a}$ & $8.57 c$ & $5.46 \mathrm{a}$ \\
\hline CHO1000 & $7.75 b$ & $7.82 \mathrm{a}$ & $7.41 \mathrm{ab}$ & $8.68 \mathrm{a}$ & $2.61 \mathrm{~b}$ \\
\hline$\mu$ & 7.44 & 7.59 & 7.35 & 8.59 & 4.23 \\
\hline \multicolumn{6}{|l|}{ Día 7} \\
\hline $\mathrm{CHOO}$ & $10.08 \mathrm{a}$ & $7.32 \mathrm{a}$ & $10.38 \mathrm{a}$ & $9.72 b$ & $5.59 \mathrm{a}$ \\
\hline $\mathrm{CHO} 200$ & $9.89 a$ & 7.04ab & $10.25 a$ & $9.96 a$ & $4.41 \mathrm{a}$ \\
\hline $\mathrm{CHO} 400$ & $10.07 \mathrm{a}$ & $7.26 \mathrm{a}$ & $10.11 a$ & $9.92 \mathrm{a}$ & $4.73 a$ \\
\hline $\mathrm{CHO} 600$ & $9.62 b$ & 7.17ab & $10.27 a$ & $10.01 \mathrm{a}$ & $4.47 \mathrm{a}$ \\
\hline CHO800 & $9.87 \mathrm{a}$ & $6.78 b$ & $10.28 a$ & $9.91 \mathrm{a}$ & $4.60 \mathrm{a}$ \\
\hline CHO1000 & $9.89 a$ & $7.27 a$ & $10.09 a$ & $9.89 a$ & $5.40 \mathrm{a}$ \\
\hline EEM & 0.03 & 0.10 & 0.05 & 0.02 & 0.53 \\
\hline$\mu$ & 9.90 & 7.14 & 10.23 & 9.90 & 4.07 \\
\hline \multicolumn{6}{|l|}{ P-value } \\
\hline $\mathrm{Ti}_{\mathrm{i}}$ & $<0.0001$ & 0.0003 & 0.0021 & $<0.0001$ & 0.0181 \\
\hline$\delta j$ & $<0.0001$ & $<0.0001$ & $<0.0001$ & $<0.0001$ & 0.0500 \\
\hline$(\mathrm{T} \delta) \mathrm{ij}$ & $<0.0001$ & 0.0376 & 0.0137 & 0.0101 & 0.0499 \\
\hline
\end{tabular}

${ }^{1}$ Tratamientos $\mathrm{CHO}$ = chorizo con carne control (dieta sin AEO); $\mathrm{CHO} 200$ = chorizo con carne de pollo suplementado con $200 \mathrm{mg}$ kg ${ }^{-1} \mathrm{AEO}$ en dieta; $\mathrm{CHO} 400$ $=$ chorizo con carne de pollo suplementado con $400 \mathrm{mg} \mathrm{kg}^{-1} \mathrm{AEO}$ en dieta; $\mathrm{CHO} 600=$ chorizo con carne de pollo suplementado con $600 \mathrm{mg} \mathrm{kg}{ }^{-1} \mathrm{AEO}$ en dieta; $\mathrm{CHO} 800$ = chorizo con carne de pollo suplementado con $800 \mathrm{mg} \mathrm{kg}^{-1}$ AEO en dieta, y CHO1000 = chorizo con carne de pollo suplementado con $1000 \mathrm{mg}$ $\mathrm{kg}^{-1} \mathrm{AEO}$ en dieta; $\mathrm{EEM}=$ error estándar de medias; $\mu=$ media global; $\tau_{\mathrm{i}}=$ efecto del i-esimo tratamiento; $\delta_{\mathrm{j}}=$ efecto del j-esimo día de evaluación $(1, \mathrm{y} 7 \mathrm{~d}$ ); $(T \delta)_{i j}=$ efecto de la interacción entre el i-esimo tratamiento y el j-esimo día.

${ }^{2} \mathrm{UFC}=$ unidades formadoras de colonias; $\mathrm{BAL}=$ bacterias ácido lácticas.

${ }^{3} \mathrm{CA}=$ capacidad antioxidante.

a-e Medias $(n=6)$ dentro de la misma columna y para diferentes tratamientos en cada día con diferente superíndice difieren significativamente cuando el p-value de $(T \delta)_{i j}<0.05$. 
para $\mathrm{CHO0}$. Caso contrario ocurrió en las BAL: $\mathrm{CHO} 0$ resultó tener el menor conteo y los tratamientos con AEO (CHO200-CHO1000) incrementaron el conteo de las BAL. González y Díez (2002) obtuvieron comportamientos similares en mesófilos aerobios y BAL durante el proceso de maduración de chorizo durante 66 días. Estos resultados podrían indicar que la población de BAL se ve favorecida en los chorizos elaborados con carne de pollo suplementados con AEO en las dietas, ya que mostraron las cuentas más elevadas. Por otro lado, Casquete et al. (2012) encontraron comportamientos similares en los conteos de mesófilos, pero contrastan con los coliformes, porque fueron más bajos en salchichón y chorizo. Sin embargo, estos autores discuten que técnicas de biopreservación han sido aplicadas para productos cárnicos e involucran la incorporación de microflora competitiva de BAL como cultivos protectores. En esta investigación, el conteo de BAL se incrementó para el día 7, por lo que podríamos suponer que en todos los tratamientos se tienen condiciones para el crecimiento adecuado de las bacterias encargadas de la fermentación (maduración). Los extractos de semilla de uva y castaña incrementaron las BAL a los 50 días (Lorenzo et al. 2013); similares resultados se obtuvieron en el chorizo a los 7 días. Los efectos encontrados por Lorenzo et al. (2013) y esta investigación indican que los extractos naturales con actividad antioxidante incrementan las BAL durante el proceso de maduración.

La capacidad antioxidante (CA) para el día 1 mejoró para $\mathrm{CHO}$ y $\mathrm{CHO} 800$, pero no para $\mathrm{CHO} 600$ ni para $\mathrm{CHO} 1000$, mientras que para el día 7 no hubo diferencias $(p>0.05)$ en la capacidad antioxidante (Cuadro 3). Broncano et al. (2011) indicaron que la actividad antioxidante en extractos de salchichas fermentadas puede ser atribuida a la proteólisis de los productos formados durante la elaboración. En este sentido, los resultados obtenidos en esta investigación con carne de pollo podrían indicar que la proteólisis del producto es retardada por la acción del AEO, ya que las moléculas de timol y carvacrol presentes en el AEO están en la pasta del chorizo. De esta forma, la degradación de las proteínas miofibrilares y sarcoplásmicas durante la fermentación y maduración pudo haberse controlado, y, en consecuencia, la formación de péptidos y aminoácidos libres (Broncano et al. 2011; Zhang et al. 2010). Resultados en CA más altos fueron obtenidos por Broncano et al. (2012), cuando identificaron componentes antioxidantes de chorizos durante 20 días de maduración, por lo que esto pudo ser la diferencia detectada de chorizos para el día 7. Esto evitó una degradación prolongada del sarcómero, y por lo tanto menos aminoácidos libres.

En este estudio, se mostró que en el tiempo, los mesófilos, coliformes y BAL aumentaron en UFC, pero los psicrótrofos y la CA decrecieron; este resultado podría deberse a los procesos de degradación de los nutrimentos de los alimentos, debido a la protéolisis y lipólisis, que generaron las condiciones para el crecimiento de algunas bacterias sin que las afectara el origen de la carne de pollos que se suplementaron con AEO durante su engorda.

\section{Evaluación SENSORIAL}

La evaluación sensorial de los alimentos mediante pruebas afectivas proporciona información sobre el grado de aceptabilidad de nuevos productos o en desarrollo. En la evaluación de los chorizos con carne de pollo existieron diferencias estadísticas $(\mathrm{p}<0.05)$ entre los días de evaluación $(\delta j)$. Así, el color rojo, el olor, el sabor y la textura obtuvieron una mejor preferencia el día 1 (Figura 1) que el día 7 (Figura 2). Sin embargo, los días 1 y 7 los chorizos con AEO presentaron mayor preferencia $(>4.50)$ respecto al control $(\mathrm{CHO})$ en color, sabor y textura. Cuando evaluaron antioxidantes naturales (extracto de semilla de uva y castaña) en chorizos, Lorenzo et al. (2013) no encontraron diferencias estadísticas en color, aroma, sabor rancio y anormal, pero sí en aceptabilidad de la textura.

En la aceptabilidad global, los chorizos fueron más aceptables $(\mathrm{p}<0.05)$ el día 1 (Figura 1$)$ que el día 7 (Figura 2). No obstante, en general, los chorizos elaborados con carne de pollo suplementados con AEO obtuvieron mejor aceptabilidad (> 5.10). Martín-Sánchez et al. (2011) evaluaron en un producto fermentado curado (salchichón) el efecto de AEO en la superficie, para encontrar pocas diferencias estadísticas en dureza. Esto contrasta con los resultados de este estudio en chorizos con carne de pollo, donde todos los atributos sensoriales fueron diferentes entre los días de evaluación. Estos autores indicaron que el AEO podría ser usado para reducir el tiempo de maduración 


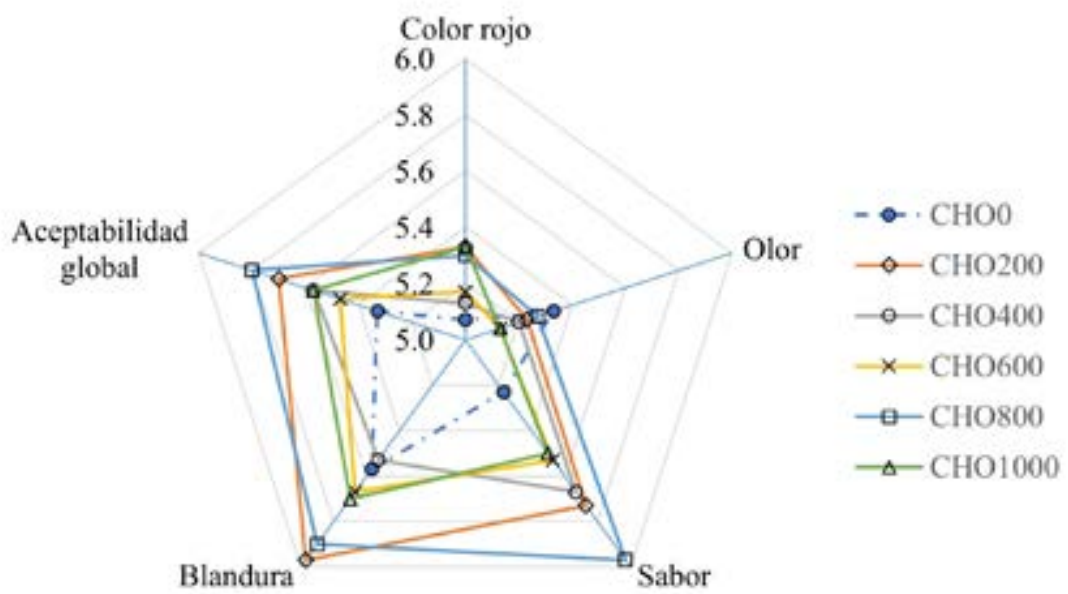

Figura 1. Atributos sensoriales de chorizos de carne de pollo suplementados con aceite esencial de orégano al día 1 almacenado a $4{ }^{\circ} \mathrm{C}$. ${ }^{1}$ Tratamientos $\mathrm{CHO0}=$ chorizo con carne control (dieta sin AEO); $\mathrm{CHO} 200=$ chorizo con carne de pollo

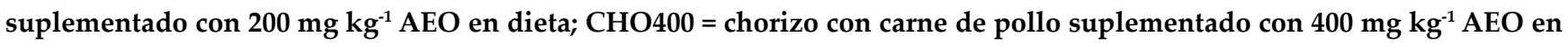
dieta; $\mathrm{CHO} 600$ = chorizo con carne de pollo suplementado con $600 \mathrm{mg} \mathrm{kg}^{-1}$ AEO en dieta; CHO800 = chorizo con carne de pollo suplementado con $800 \mathrm{mg} \mathrm{kg}^{-1} \mathrm{AEO}$ en dieta, y CHO1000 = chorizo con carne de pollo suplementado con $1000 \mathrm{mg}^{\mathrm{k}}^{-1}$ AEO en dieta.

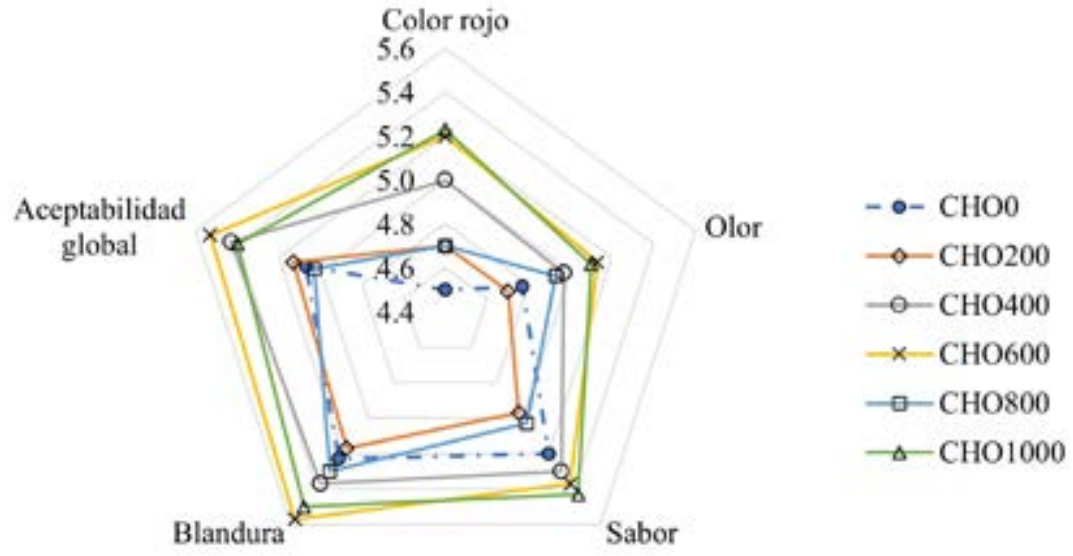

Figura 2. Atributos sensoriales de chorizos de carne de pollo suplementados con aceite esencial de orégano al día 7 almacenados a $4{ }^{\circ} \mathrm{C}$. ${ }^{1}$ Tratamientos $\mathrm{CHOO}=$ chorizo con carne control (dieta sin AEO); $\mathrm{CHO} 200=$ chorizo con carne de pollo

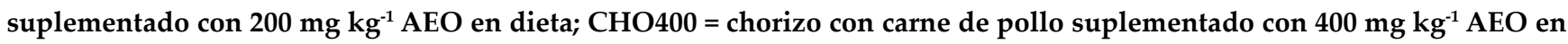
dieta; $\mathrm{CHO} 600$ = chorizo con carne de pollo suplementado con $600 \mathrm{mg} \mathrm{kg}^{-1}$ AEO en dieta; CHO800= chorizo con carne de pollo suplementado con $800 \mathrm{mg} \mathrm{kg}^{-1} \mathrm{AEO}$ en dieta, y CHO1000 = chorizo con carne de pollo suplementado con $1000 \mathrm{mg}^{\mathrm{kg}} \mathrm{C}^{-1}$ AEO en dieta. 
y mejorar las propiedades sensoriales. Las diferencias encontradas en los días de los atributos sensoriales en los chorizos pueden deberse a lo indicado por Ruiz-Capillas et al. (2012), la característica del gusto de las salchichas fermentadas está influenciada por la fuente, la cantidad, el tipo de ingredientes y las condiciones de proceso.

\section{CONCLusiones}

El chorizo elaborado con pechugas de pollos suplementados con aceite esencial de orégano en las dietas mejoró el $\mathrm{pH}$. Los parámetros de color fueron mejores en 200 (CHO200) y $1000 \mathrm{mg} \mathrm{kg}^{-1}$ (CHO1000). El cambio de color total disminuyó en los chorizos elaborados con la carne de pollos suplementados con las concentraciones más altas de aceite esencial de orégano $\left(400,600,800\right.$ y $\left.1000 \mathrm{mg} \mathrm{kg}^{-1}\right)$.

Los chorizos CHO800 y CHO1000 (800 y 1000 $\mathrm{mg} \mathrm{kg}{ }^{-1}$ de AEO en dieta) incrementaron la fuerza de corte y dureza. El conteo de bacterias ácido lácticas se incrementó en los chorizos elaborados con carne de pollos suplementados con las concentraciones más altas de aceite de orégano. Los atributos sensoriales fueron más preferidos en el primer día de evaluación; no obstante, un perfil sensorial podría proporcionar resultados más acertados sobre atributos más específicos como el aroma.

Esta investigación sugiere la necesidad de desarrollar proyectos dirigidos a la producción primaria sin aditivos sintéticos, y su utilidad como materia prima para elaborar productos alimenticios sin conservadores químicos. 


\section{LITERATURA CITADA}

Arcila-Lozano CC, Loarca-Piña G, Lecona-Uribe S, González de Mejía E. 2004. El orégano: propiedades, composición y actividad biológica de sus componentes. Archivos Latinoamericanos de Nutrición 54: 100-111.

Bozkurt H, Bayram M. 2006. Colour and textural attributes of sucuk during ripening. Meat Science 73: 344350. https://doi.org/10.1016/j.meatsci.2006.01.001

Broncano JM, Timón ML, Parra V, Andrés AI, Petrón MJ. 2011. Use of proteases to improve oxidative stability of fermented sausages by increasinglow molecular weight compounds with antioxidant activity. Food Research International 44: 2655-2659. https://doi. org/10.1016/j.foodres.2011.05.011

Broncano JM, Otte J, Petrón MJ, Parra V, Timón ML. 2012. Isolation and identification of low molecular weight antioxidant compounds from fermented "chorizo" sausages. Meat Science 90: 494-501. https://doi. org/10.1016/j.meatsci.2011.09.015

Burt S. 2004. Essential oils: their antibacterial properties and potential applications in foods-a review. International Journal of Food Microbiology 94: 223253. https://doi.org/10.1016/j.ijfoodmicro.2004.03.022

Cantú-Valdéz JA, Gutiérrez-Soto G, Hernández-Martínez CA, Sinagawa-García SR, Quintero-Ramos A, Hume ME, Herrera-Balandrano DD, Méndez-Zamora G. 2020. Mexican oregano essential oils as alternatives to butylated hydroxytoluene to improve the shelf life of ground beef. Food Science \& Nutrition 8:4555-4564. https://doi.org/10.1002/fsn3.1767

Casquete R, Benito MJ, Martín A, Ruiz-Moyano S, Aranda E, Córdoba MG. 2012. Microbiological quality of salchichon and chorizo, traditional Iberian dry-fermented sausages from two different industries, inoculated with autochthonous starter cultures. Food Control 24: 191-198. https://doi.org/10.1016/j.foodcont.2011.09.026

Cázares-Gallegos R, Silva-Vázquez R, HernándezMartínez CA, Gutiérrez-Soto JG, Kawas-Garza JR, Hume ME, Méndez-Zamora G. 2019. Performance, carcass variables, and meat quality in broilers supplemented with dietary Mexican oregano oil. Brazilian Journal of Poultry Science 21: 1-10. https://doi. org/10.1590/1806-9061-2018-0801

Charles JC, González CG, Herrera DD, Hernández CA, Gutiérrez G, Silva R, Hume ME, Méndez G.
2019. Oregano essential oil as vinegar substitute in pork chorizo formulation. Revista Chapingo Serie Zonas Áridas 18(2): 11-25. https://doi.org/10.5154/r. rchsza.2018.08.017

Corral S, Salvador A, Flores M. 2013. Salt reduction in slow fermented sausages affects the generation of aroma active compounds. Meat Science 93: 776-785. https://doi.org/10.1016/j.meatsci.2012.11.040

Dorman H, Peltoketo A, Hiltunen R, Tikkanen MJ. 2003. Characterization of the antioxidant properties of deodourised aqueous extracts from selected Lamiaceae herbs. Food Chemistry 83: 255-262. https://doi. org/10.1016/S0308-8146(03)00088-8

Fasseas MK, Mountzouris KC, Tarantilis PA, Polissiou M, Zervas G. 2007. Antioxidant activity in meat treated with oregano and sage essential oils. Food Chemistry 106: 1188-1194. https://doi.org/10.1016/j. foodchem.2007.07.060

González B, Díez V. 2002. The effect of nitrite and starter culture on microbiological quality of "chorizo"-a Spanish dry cured sausage. Meat Science 60: 295-298. https://doi.org/10.1016/S0309-1740(01)00137-1

Hernández-Coronado AC, Silva-Vázquez R, Rangel-Nava ZE, Hernández-Martínez CA, Kawas-Garza JR, Hume ME, Méndez-Zamora G. 2019. Mexican oregano essential oils given in drinking water on performance, carcass traits, and meat quality of broilers. Poultry Science 98: 3050-3058. https://doi.org/10.3382/ps/pez094

Herrera-Balandrano DD, Martínez-Rojas D, LunaMaldonado AI, Gutiérrez-Soto G, Hernández-Martínez CA, Silva-Vázquez R, Flores-Girón E, QuinteroRamos A, Méndez-Zamora G. 2020. Conservación de pechugas de pollo con aceite esencial de orégano mexicano. Biotecnia 22: 119-127. https://doi.org/10.18633/ biotecnia.v22i2.1253

Hung Y, de Kok TM, Verbeke W. 2016. Consumer attitude and purchase intention towards processed meat products with natural compounds and a reduced level of nitrite. Meat Science 121: 119-126. https://doi. org/10.1016/j.meatsci.2016.06.002

Hygreeva D, Pandey MC, Radhakrishna K. 2014. Potential applications of plant based derivatives as fat replacers, antioxidants and antimicrobials in fresh and processed meat products. Meat Science 98: 47-57. https:// doi.org/10.1016/j.meatsci.2014.04.006

Jiang J, Xiong YL. 2016. Natural antioxidants as food and feed additives to promote health benefits and quality 
of meat products: a review. Meat Science 120: 107-117. https://doi.org/10.1016/j.meatsci.2016.04.005

Ledesma E, Laca A, Rendueles M, Díaz M. 2016. Texture, colour and optical characteristics of a meat product depending on smoking time and casing type. LWTFood Science and Technology 65: 164-172. https://doi. org/10.1016/j.lwt.2015.07.077

Lorenzo JM, González-Rodríguez RM, Sánchez M, Amado IR, Franco D. 2013. Effects of natural (grape seed and chestnut extract) and synthetic antioxidants (buthylatedhydroxytoluene, BHT) on the physical, chemical, microbiological and sensory characteristics of dry cured sausage "chorizo". Food Research International 54: 611-620. https://doi.org/10.1016/j. foodres.2013.07.064

Martín-Sánchez AA, Chaves-López C, Sendra E, Sayas E, Fenández-López J, Pérez-Álvarez JÁ. 2011. Lipolysis, proteolysis and sensory characteristics of a Spanish fermented dry-cured meat product (salchichón) with oregano essential oil used as surface mold inhibitor. Meat Science 89: 35-44. https://doi.org/10.1016/j. meatsci.2011.03.018

Meilgaard M, Civille GV, Carr TB. 2006. Affective tests consumer tests and in-house panel acceptance tests. In: Meilgaard M, Civille GV, Carr TB, editors. Sensory Evaluation Techniques. Boca Ratón, CRC Press. P. 231-251.

Menegas LZ, Colombo Pimentel T, Garcia S, Prudencio SH. 2013. Dry-fermented chicken sausage produced with inulin and corn oil: Physicochemical, microbiological, and textural characteristics and acceptability during storage. Meat Science 93: 501-506. https://doi. org/10.1016/j.meatsci.2012.11.003

Mielnik MB, Aaby K, Skrede G. 2003. Commercial antioxidants control lipid oxidation in mechanically deboned turkey meat. Meat Science 65: 1147-1155. https://doi. org/10.1016/S0309-1740(02)00345-5

Mora-Gallego H, Dolors Guàrdia M, Serra X, Gou P, Arnau J. 2016. Sensory characterization and consumer acceptability of potassium chloride and sunflower oil addition in small-caliber non-acid fermented sausages with a reduced content of sodium chloride and fat. Meat Science 112: 9-15. https://doi.org/10.1016/j. meatsci.2015.10.008

Perales-Jasso YJ, Gamez-Noyola SA, Aranda-Ruiz J, Hernandez-Martinez CA, Gutierrez-Soto G, LunaMaldonado AI, Silva-Vázquez R, Hume ME, Mendez-
Zamora G. 2018. Oregano powder substitution and shelf life in pork chorizo using Mexican oregano essential oil. Food Science \& Nutrition 6(5): 1254-1260. https://doi.org/10.1002/fsn3.668

Radulović Z, Živković D, Mirković N, Petrušić M, Stajić S, Perunović M, Paunović D. 2011. Effect of probiotic bacteria on chemical composition and sensory quality of fermented sausages. Procedia Food Science 1: 1516-1522. https://doi.org/10.1016/j. profoo.2011.09.224

Ruiz-Capillas C, Triki M, Herrero AM, Rodriguez-Salas L, Jiménez-Colmenero F. 2012. Konjac gel as pork backfat replacer in dry fermented sausages: Processing and quality characteristics. Meat Science 92: 144-150. https://doi.org/10.1016/j.meatsci.2012.04.028

[SAGARPA] Secretaría de Agricultura, Ganadería, Desarrollo Rural, Pesca y Alimentación. [internet]. 2015. Norma Oficial Mexicana NOM-033-SAG/ ZOO-2014, Métodos para dar muerte a los animales domésticos y silvestres. Diario Oficial de la Federación. Distrito Federal, México. [cited 2020 Jul 6]. Disponible en: https://www.dof.gob.mx/nota_detalle.php?codigo $=5405210 \&$ fecha $=26 / 08 / 2015$

Sánchez-Zamora N, Silva-Vázquez R, Rangel-Nava ZE, Hernández-Martínez CA, Kawas-Garza JR, Hume ME, Herrera-Balandrano DD, Méndez-Zamora G. 2019. Inulina de agave y aceite de orégano mejoran la productividad de pollos de engorda. Ecosistemas y Recursos Agropecuarios 6(18): 523-534. https://doi. org/10.19136/era.a6n18.2197

[SAS] Statistical Analysis System Institute. 2006. SAS, User's GuideVers. 9.1. SAS Institute Inc. Cary, USA. Silva-Vázquez R, Duran-Meléndez LA, HernándezMartínez CA, Gutiérrez-Soto JG, Hume ME, MéndezZamora G. 2018. Effects of two sources of Mexican oregano oil on performance, blood profile, carcass variables, and meat of broilers. Revista Brasileira de Zootecnia 47: e20170198. https://doi.org/10.1590/ rbz4720170198

[SS] Secretaría de Salud. [internet]. 1995. Norma Oficial Mexicana NOM-110-SSA1-1994, Bienes y servicios. Preparación y dilución de muestras de alimentos para su análisis microbiológico. [cited 2019 Dic 16]. Disponible en: http://www.economia-noms.gob.mx/ noms/inicio.do

Vazquez SR, Dunford NT. 2005. Bioactive components of Mexican Oregano oil as affected by moisture and 
plant growth. Journal of Essential Oil Research 17: 668671. https://doi.org/10.1080/10412905.2005.9699028

Wood JD, Richardson RI, Nute GR, Fisher AV, Campo MM, Kasapidou E, Sheard PR, Enser M. 2003. Effects of fatty acids on meat quality: a review. Meat Science 66: 21-32. https://doi.org/10.1016/S0309-1740(03)00022-6

Zhang W, Xiao S, Samaraweera H, Lee EJ, Ahn DU. 2010. Improving functional value of meat products. Meat Science 86: 15-31. https://doi.org/10.1016/j. meatsci.2010.04.018 